\title{
Impacts of Bacillus subtilis JQ61816 on lipid panel and expression of genes involved in cholesterol metabolism in hypercholesterolemic rats
}

\author{
Abolfazl Dehkohneh, Azam Khalili, Omid Ardalani², Amin Mohsenzadeh33, Hoda Sabati4, Parvaneh Jafari5,* \\ ${ }^{1}$ Department of Biotechnology, Faculty of Advanced Science and Technology, Tehran Medical Sciences, Islamic Azad University, \\ Tehran, Iran \\ 2Department of Microbial Biotechnology, School of Biology and Centre of Excellence in Phylogeny of Living Organisms, College of \\ Science, University of Tehran, Tehran, Iran \\ 3Department of Microbiology, Faculty of Science, Ardabil branch, Islamic Azad University, Ardabil, Iran \\ ${ }_{4}^{4}$ Biotechnology and Biological Science Research Center, Faculty of Science, Shahid Chamran University of Ahvaz, Ahvaz, Iran \\ ${ }_{5}$ Microbiology Department, Faculty of Science, Islamic Azad University, Arak Branch, Arak, Iran
}

\begin{abstract}
Hypercholesterolemia is one of the major risk factors associated with the emergence and development of cardiovascular diseases (CVD) and atherosclerosis. The hypocholesterolemic effects of probiotics have been indicated by numerous studies. The 3-hydroxy-3-methylglutaryl-coenzyme A reductase (HMGCR) and cytochrome P450 7A1 or cholesterol 7 alpha-hydroxylase (CYP7A1) are two important genes in choletsterol metabolism. In this study, the effects of Bacillus subtilis JQ61816 on lipid panel, hepatic enzymes and expression levels of HMGCR and CYP7A1 were investigated. Twenty-one male Wistar rats were randomly allotted to 3 experimental groups; a) negative control group (ND) fed with normal diet, b) high-fat diet group (HFD) fed with high cholesterol diet, and c) probiotic group (BS) fed with high cholesterol diet supplemented with probiotic $B$. subtilis. Serum analysis of treatment groups was performed to measure fasting blood sugar (FBS), lipid profile parameters, hepatic enzymes, urea, and uric acid. Our results showed that $B$. subtilis could reduce the level of total cholesterol, triglycerides, and LDL and it also could increase high-density lipoprotein (HDL) level. Moreover, alanine transaminase (ALT), aspartate transaminase (AST), and uric acid were significantly lower in BS group compare to HFD group. Furthermore, up-regulation of HMGCR and down-regulation of CYP7A1 were observed in BS group. The results of our study suggest that consumption of probiotic B. subtilis JQ61816 may prevent or decline the development of hypercholesterolemia and other cardiovascular diseases.
\end{abstract}

Keywords: Probiotics, Bacillus subtilis, Hypercholesterolemia, Cardiovascular disease, Triglyceride

\section{Introduction}

Hypercholesterolemia is one of the main risk factors associated with the emergence and development of cardiovascular diseases (CVD) and

\footnotetext{
*Corresponding author:

Parvaneh Jafari, Ph.D

Microbiology Department, Faculty of Science, Islamic Azad University, Arak Branch, Arak, Iran

Tel/Fax: +98 8634132451

Email: p-jafari@iau-arak.ac.ir, Parvaneh.jafari@gmail.com

http://orcid.org/oooo-0003-2771-9309
}

Received: July, 23, 2021

Accepted: September, 22, 2021 atherosclerosis, which are considered as common cause of morbidity and mortality [1]. According to world health organization (WHO) prediction, by 2030, CVD will be the leading global cause of deaths (c) The Author(s) 2021
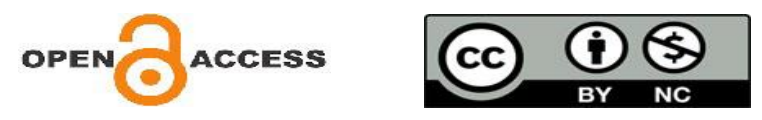
and will affect over 23.6 million around the world which accounts for $80 \%$ of the deaths in low-income countries $[2,3]$.

There are several approaches used to control or treat hypercholesterolemia, such as consumption of Statins and Ezetimibe, exercising and adjusting of dietary habits [4-6]. Although medicinal therapy is a pioneering method in the regulation of cholesterol level, long-term use of this approach leads to various undesirable effects [7-9]. Thus, there has been a growing tendency among researchers to find alternative ways, such as using biomaterials.

Numerous studies have indicated that probiotics, either bacteria or yeasts, have many health-promoting effects such as immunomodulatory, anti-cancer and anti-diabetic, gastrointestinal tract promotion, hypocholesterolemic and regulation of lipid metabolism [10-14]. For these reasons, probiotics are considered as a promising approach in the modulation of lipid metabolism to reduce the rate of hypercholesterolemia. Probiotics are living microorganisms that exert their beneficial effects on the host if consumed in appropriate amounts. Bacillus subtilis is a type of probiotics which is widely used in diets [15].

In the present study, the impact of oral administration of $B$. subtilis JQ61816 on lipid panel parameters and the expression levels of 3-hydroxy-3methyl-glutaryl-coenzymeA reductase (HMGCR) and cholesterol 7-alpha hydroxylase (CYP7A1) genes were assessed in male Wistar rats with a high-fat diet.

\section{Materials and Methods}

\subsection{Bacteria}

The B. Subtilis JQ61816 was purchased from Takgen company as lyophilized bacterium (Bactogen ${ }^{\circledR}$ contained $4 \times 10^{9} \mathrm{CFU} / \mathrm{ml}$ ). Probiotic suspension daily prepared, and to prepare probiotic suspension, lyophilized B. Subtilis JQ61816 mixed by phosphatebuffered saline (PBS) (Sigma Aldrich, USA).

\subsection{Animals and study design}

All animal experiments in this study were approved by the Animal Care Committee of Islamic Azad University of Medical Science (Tehran, Iran) and were performed according to the guidelines from the NIH principles for the Care and Use of Laboratory Animals. Twenty-one male Wistar rats aged 7-8 weeks; weighed 100-150 grams were purchased from
Baqiyat-Allah Research Center (Tehran, Iran), and used for all experiments. Rats were housed in cages maintained at $22 \pm 2^{\circ} \mathrm{C}$ with $55 \% \pm 5 \%$ humidity and subjected to a $12 \mathrm{~h}$ light/dark cycle and free access to food and water for 14 days to adapt to laboratory conditions after adaptation rats were distributed into three groups. Negative control group (ND), High-fat group (HFD), and probiotic group (BS), which respectively were fed with normal diet plus $1 \mathrm{ml}$ PBS, high-fat diet $+1 \mathrm{ml} \mathrm{PBS}$, and high cholesterol diet $+B$. subtilis $\left(4 \times 10^{9} \mathrm{CFU} / \mathrm{ml}\right)$ suspension. Diet ingredients are shown in Table 1. To prepare a high-fat diet, we used liquid fat and mixed it with the normal diet's ingredients. PBS and probiotic suspension were administered by daily gavage at a certain time.

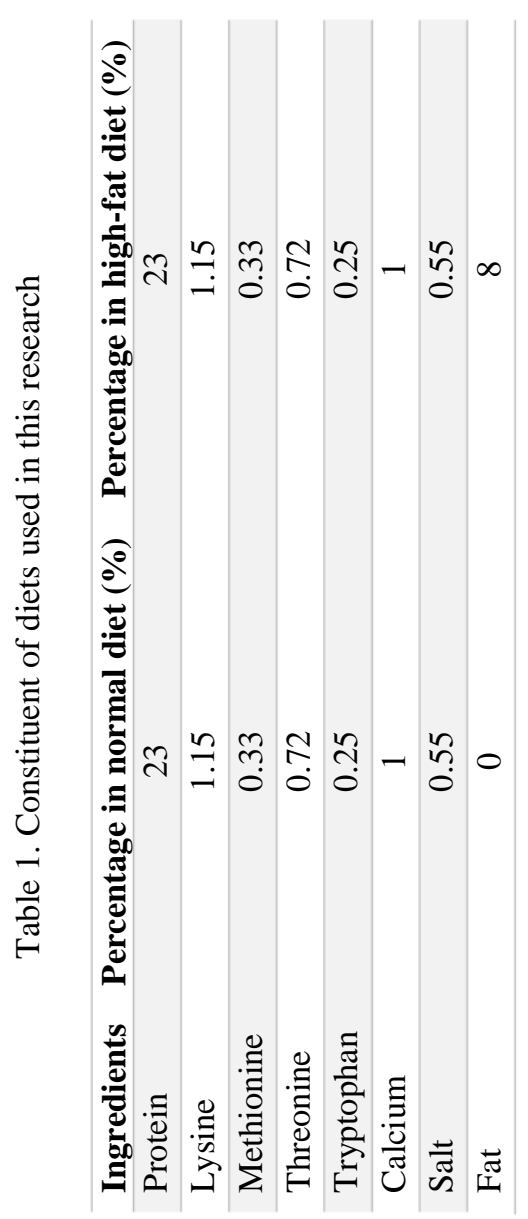

\subsection{Blood collecting and analysis}

At the end of three weeks, all rats were sacrificed after $12 \mathrm{~h}$ fasting, and then blood was collected by cardiac puncture. The serum was collected by centrifugation at $1500 \times g$ for $15 \mathrm{~min}$. An auto-analyzer 
(RA10oo, USA) was used for the analysis of serum total cholesterol (TC), triglyceride (TG), low-density lipoprotein (LDL), high-density lipoprotein (HDL), aspartate aminotransferase (AST), alkaline phosphatase (ALP), alanine aminotransferase (ALT), fasting blood sugar (FBS), urea and uric acid levels. Moreover, to achieve a better understanding of the impact of probiotics on serum parameters, we calculated the ratio of total cholesterol/HDL, triglycerides/HDL, and LDL/HDL.

\subsection{Gene expression analysis}

Total RNA was extracted from abdominal adipose tissues using RNX-Plus reagent (Cinaclon, Iran) according to manufacturer's instruction, and $1 \mu \mathrm{g}$ of isolated RNA was converted to cDNA with cDNA Reverse Transcriptase Kit (Bioneer, South Korea). cDNA was used as a template and amplified by quantitative polymerase chain reaction (qPCR). Gene expression analysis was performed by Real-time PCR system (ABI-Stepone, USA) using SYBRGreen (Ampliqon, Denmark). The program for PCR and relative gene expression is described in our previous study [11]. GAPDH gene was considered as a housekeeping gene, and the relative quantities of each gene were analyzed using terms of $2^{-\Delta \Delta C t}$. Primers were designed for HMGCR, and CYP7A1 genes are obtained from our previous research [11]. The primers are designed using Primer3plus software and primer analysis was performed by OligoAnalyzer 3.1. Resultant primers are shown in Table 2. The whole experiments were carried out in duplicate.

\subsection{Statistical analysis}

All data are reported as the mean \pm SEM. Statistical significance was analyzed by the student ttest method using GraphPad Prism 8.2.1 Software (GraphPad Software, Inc., US). A $P$-value $<0.05$ was considered statistically significant.

\section{Results}

3.1 The effect of B. subtilis JQ61816 on FBS

HFD group had the highest level of FBS among the experimental group. Although B. subtilis JQ61816 could reduce the FBS level, there was not a significant difference compared to control groups (Figure 1A).

3.2 The effect of B. subtilisJQ61816 on lipidprofile 3.2.1 Total Cholesterol
The level of total cholesterol was significantly increased in HFD group by $22.9 \%(\mathrm{P}<0.05)$, while $B$. subtilis JQ61816 caused decreasing of total cholesterol level by $9.9 \%$ compared to HFD. However, the difference was not significant (Figure 1B).

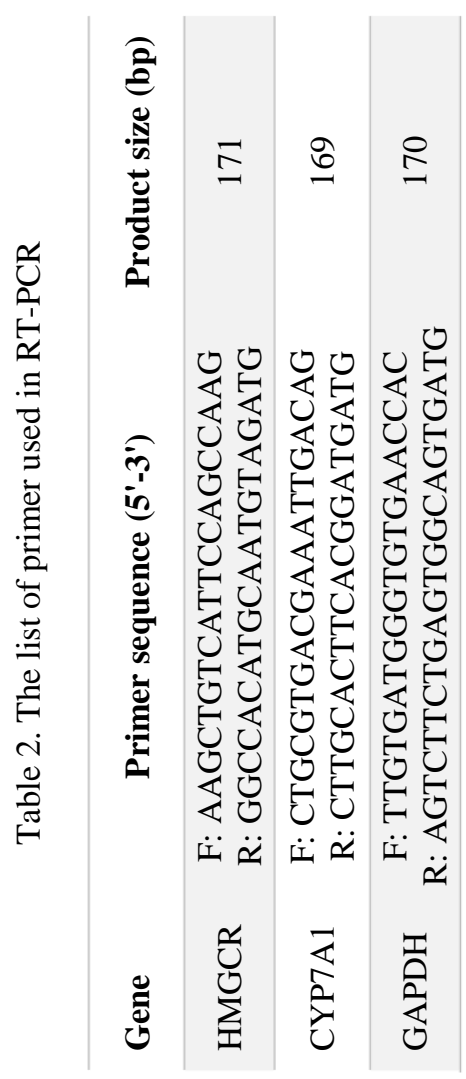

\subsubsection{Triglycerides}

The serum analysis indicated that $B$. subtilis JQ61816 consumption could significantly decrease the triglycerides level by $30.4 \%$ in BS group compared to the HFD group $(\mathrm{P}<0.05)$. The results are shown in Figure 1C.

\subsubsection{HDL}

BS group revealed the highest level of HDL among experimental groups. The level of HDL in BS was climbed by $11.76 \%$ compared to HFD group. Moreover, ND group had the lowest amount of HDL unexpectedly (Figure 1D).

\subsubsection{LDL}

As depicted in Figure 1E, HFD group had the highest level among all groups. The serum LDL was significantly decreased after 21 days of consumption of 
probiotics by $22.2 \%$ compared to HFD group other experimental groups $(\mathrm{P}<0.05)$. The result of the $(\mathrm{P}<0.05)$.
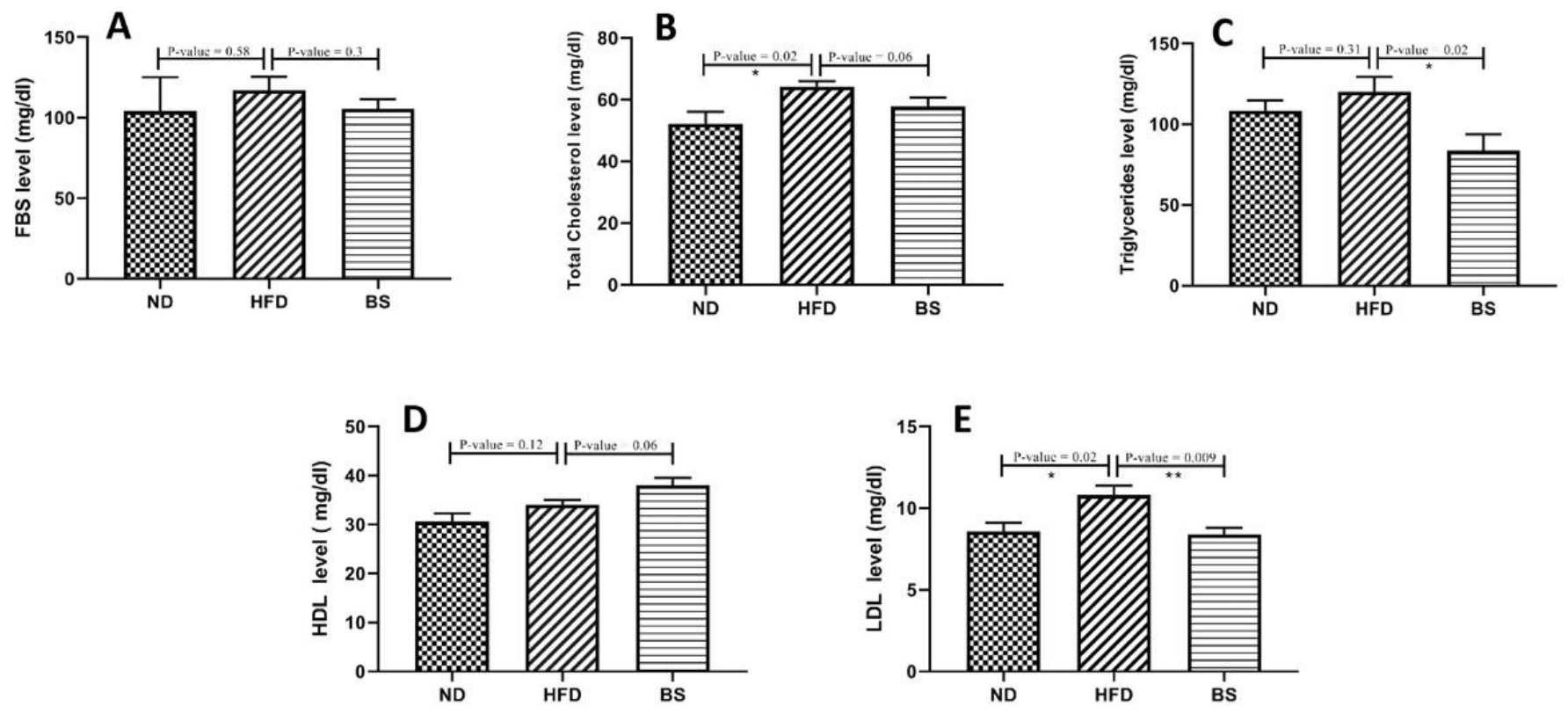

Figure1. Serum analysis results of FBS and lipid profile parameters. A) Serum FBS level: Bacillus subtilis JQ61816 could decline the level of serum FBS compared to HFD group, but it was not statistically significant; B) Total cholesterol level: the quantity of total serum cholesterol was elevated significantly by $22.9 \%$ in HFD group due to having a high-fat diet while the level of total cholesterol was reduced by $9.9 \%$ due to consumption of $B$. subtilis JQ61816; C) The level of serum triglycerides: consumption of B. subtilis JQ61816 could significantly decrease the serum triglyceride compared to HFD group; D) Serum HDL level: BS group showed The highest level of HDL, which was elevated by $11.76 \%$ compared to HFD. Unexpectedly, The ND group had the lowest level of HDL among all experimental groups; E) The serum level of LDL: BS group was significantly declined by $22.2 \%$ compared to HFD group due to consumption of probiotics.
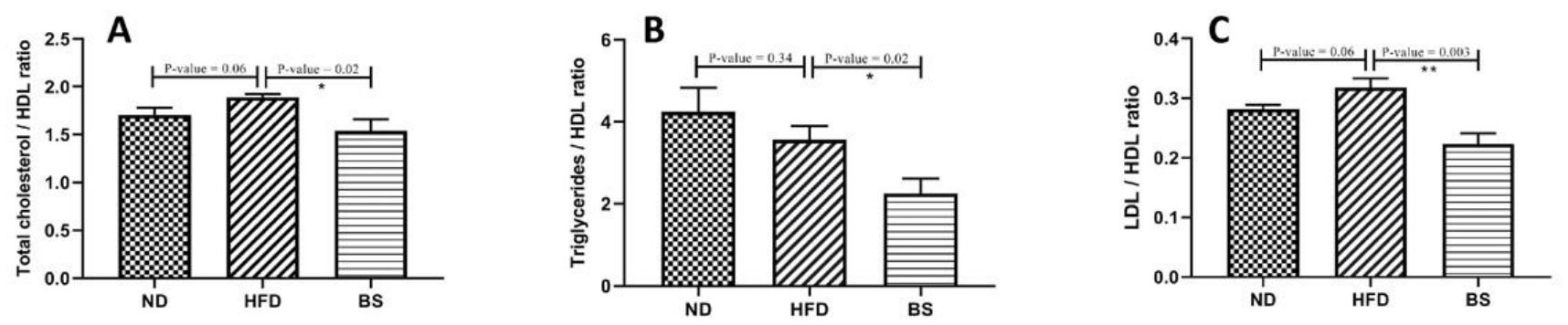

Figure 2. Ratio analysis of lipid profile parameters. A) Total cholesterol to HDL ratio: BS group is declined compared to HFD group due to consumption of $B$. subtilis JQ61816; B) Triglyceride to HDL ratio: BS group was shown a significant difference compared to HFD group by $36.8 \%$; C) LDL / HDL ratio: The highest number of LDL/HDL ratio was belonged to HFD group, while BS group had the lowest level due to probiotics consumption.

\subsubsection{Total cholesterol/HDL ratio}

As expected, the measurement of total cholesterol to HDL ratio indicated that $\mathrm{BS}$ group was lower than

\subsubsection{Triglycerides/HDL ratio}

The ratio of triglycerides number to HDL number displayed a $16.9 \%$ reduction in HFD group compared to ND group, while a statistical significance was 
observed in BS group compared to HFD group by 36.8\% $(\mathrm{P}<0.05)$ (Figure 2B).

\subsubsection{LDL/HDL ratio}

The analysis of LDL/ HDL ratio revealed that the consumption of $B$. subtilis JQ61816 was caused a significant decline compared to HFD group. The results of the analysis are shown in Figure $2 \mathrm{C}$.

\subsubsection{ALP}

The level of ALP in BS group was decreased compared to HFD. Although the amount of ALP was increased in both groups of HFD and BS compared to ND group, a significant difference was observed between HFD and ND groups $(\mathrm{P}<0.05)$. Results are shown in Figure $3 \mathrm{C}$.
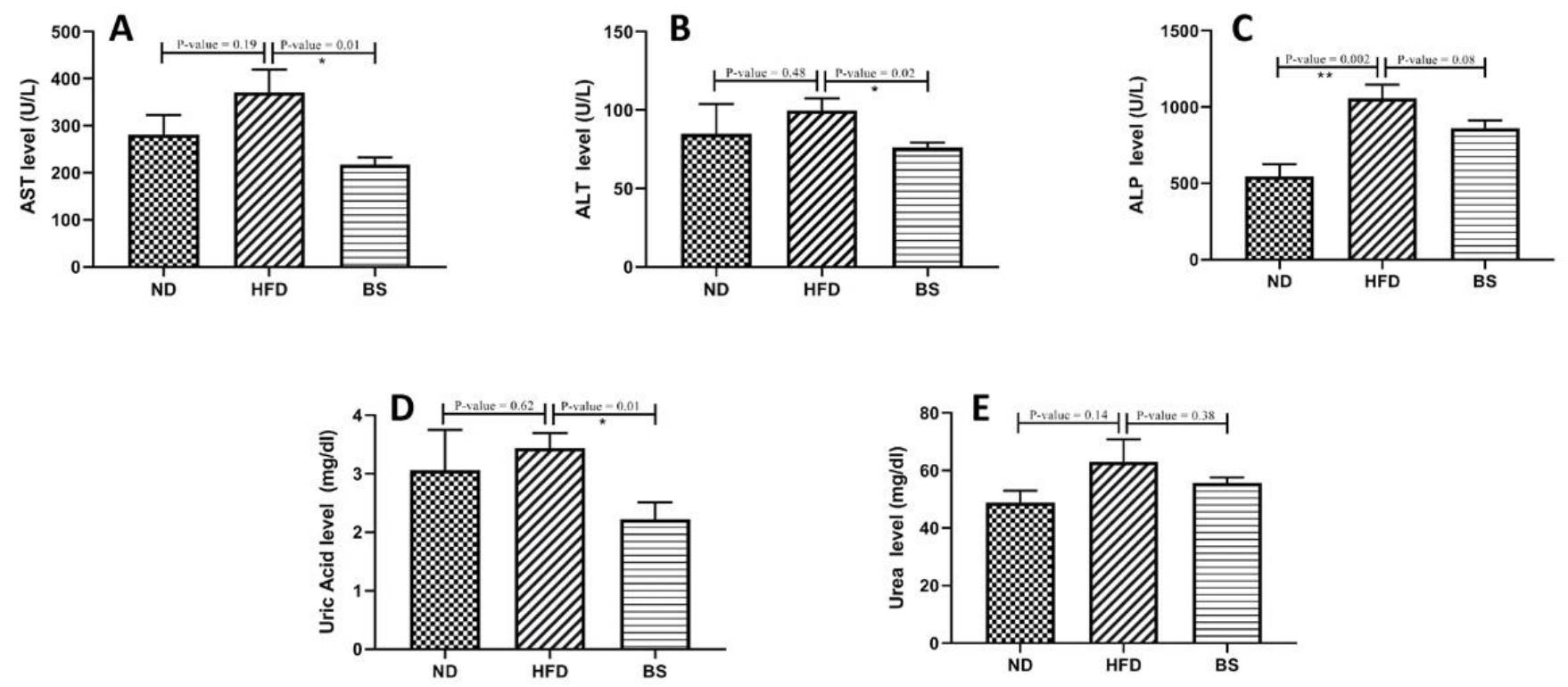

Figure 3. The level of hepatic enzymes, uric acid and urea. A) The serum levels of AST: consumption of Bacillus subtilis JQ61816 could significantly decline the amount of AST enzyme while The HFD group had the highest level of this hepatic enzyme among other experimental groups; B) The serum level of ALT: BS group had the lowest level of ALT among experimental groups and $B$. subtilis reduced the ALT level significantly compared to HFD group; C) The serum level of ALP: The amount of ALP was raised in HFD and BS groups compared to ND group. Although the BS group had a lower amount of serum ALP, there was no significant difference between HFD and BS groups; D) The serum level of uric acid: administration of probiotics was caused to reduce the uric acid level in BS group by $35.4 \%$ compared to HFD group; E) The serum level of urea: Although the level of urea in BS group is lower than HFD group, but the difference was not statistically significant.

\subsection{The effect of B. subtilis JQ61816 on hepatic} enzymes

\subsubsection{AST and ALT}

The measurement of serum AST and ALT levels revealed that the administration of probiotics could reduce these hepatic enzymes significantly compared to HFD group by $41.4 \%$ and $23.8 \%$, respectively $(\mathrm{P}<0.05)$. In contrast, the serum level of AST and ALT were climbed due to a high-fat diet (Figure 3A, $3 \mathrm{~B}$ ).

\subsection{Uric Acid}

The measurement of serum uric acid showed that orally administration of $B$. subtilis JQ61816 could reduce uric acid level by $35.4 \%$ compared to uric acid level in HFD group $(\mathrm{P}<0.05)$. The results are shown in Figure 3D.

\subsection{Urea}

The analysis of urea level demonstrated that there was no significant difference between all experimental 

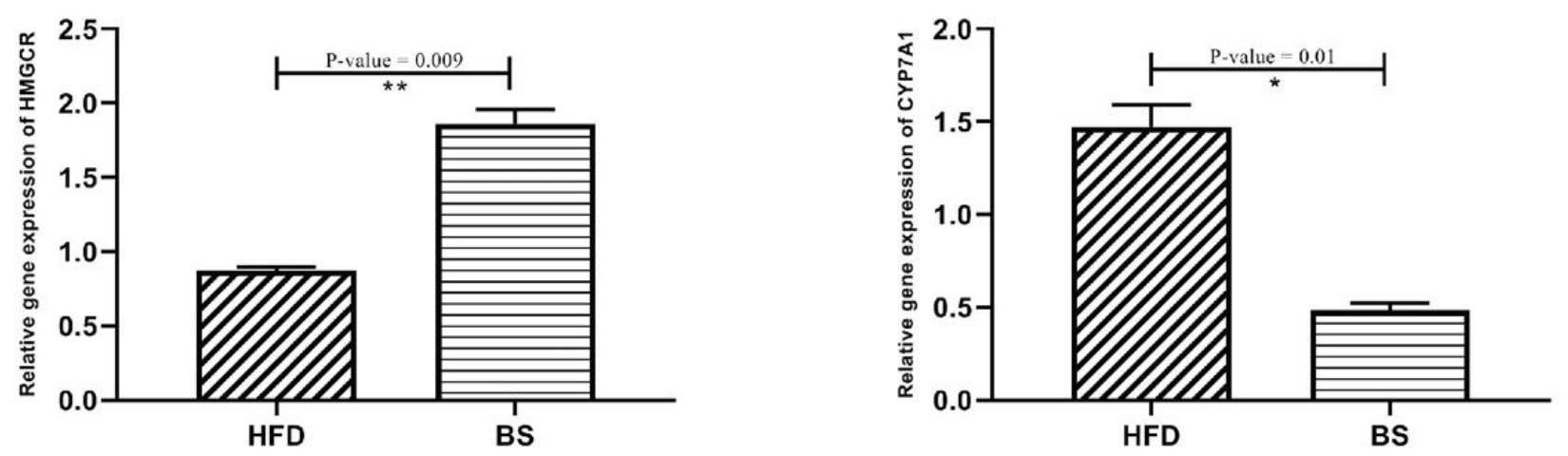

Figure 4. The effect of Bacillus subtilis JQ61816 on key genes related to cholesterol metabolism: The level of HMGCR expression was increased in BS group compared to HFD group. In contrast, unexpectedly, the level of CYP7A1 gene expression was significantly decreased in BS group due to using probiotics.

groups. However, the serum level of uric acid in BS group was lower than HFD group (Figure $3 \mathrm{E}$ ).

\subsection{The effect of B. subtilis JQ61816 on Key genes} in cholesterol metabolism

The expression of HMGCR gene was significantly climbed in BS group compared to HFD group $(\mathrm{P}<0.05)$. In contrast, the down regulation of CYP7A1 was observed in BS group. Results are shown in Figure $4 \mathrm{~A}, 4 \mathrm{~B}$.

\section{Discussion}

Hypercholesterolemia is a complex metabolic disorder caused by an elevated level of blood cholesterol and affected by lipid metabolism factors and diet. Numerous clinical, epidemiological, animal and genetic studies have indicated that a high amount of blood cholesterol is associated with an elevated risk of cardiovascular diseases [16, 17].

Using Statins (e.g., rosuvastatin and atorvastatin), as lipid-lowering medication, is the most common approach to reduce serum cholesterol, triglycerides, and LDL levels. Although this method might be effective, some undesirable effects have been reported, including myopathy and albuminuria $[18,19]$.

Hence, using alternative approaches are crucial, which are safer and more cost-effective. For a long time, probiotics have been widely used, and the hypocholesterolemic effect of them has been wellstudied in vivo and in vitro [12, 20].

In this study, we used B. subtilis JQ61816 as a probiotic. A high level of cholesterol in HFD group indicated that high-cholesterol diet had led to hypercholesterolemic rats. Our results indicated that B. subtilis JQ61816 could decrease the serum levels of total cholesterol, triglycerides, and LDL, while it can elevate HDL level. Although B. subtilis JQ61816 could reduce total cholesterol level, it was not significant statistically. The hypocholesterolemic effects of probiotics have been studied in humans and animals over the years. Similarities between humans and some animals (e.g., rats, hamsters, and pigs) in bile acid and cholesterol metabolism and plasma lipoprotein distribution make these animals as useful experimental models [21-23]. Therefore, the acquired results from animal studies can be reliable and transferable to humans. In this study, B. subtilis JQ61816 has been chosen to make a comparison with other probiotics with hypocholesterolemic effects. According to previous reports, various probiotic bacteria may have hypercholesterolemic impacts by various levels [24-26].

There are several plausible mechanisms for hypocholesterolemic effects of probiotics, including enzymatic deconjugation of bile acids by bile salt hydrolase, assimilation of cholesterol by growing cell, production of short-chain fatty acids from oligosaccharides and cholesterol binding to cellular surface of probiotics [24-26]. Also, B. subtilis bacterium can produce extracellular cholesterol oxidase, which led to cholesterol level reduction [27, 28].

Heo et al. indicated that Lactobacillus plantarum LRCC 5273 could reduce total cholesterol and LDL- 
cholesterol levels significantly, while the significant reduction in triglycerides and HDL-cholesterol were not observed [29]. In another study, Briand et al. showed that the administration of Saccharomyces boulardï led to a significant reduction in total plasma cholesterol and HDL at day 21. However, in the liver, the significant reduction was not observed in total cholesterol level, while triglycerides level significantly decreased by $25 \%$ at day 39 [30]. Moreover, according to some reports the consumption of $L$. plantarum KY1032 and Lactobacillus curvatus at $5 \times 10^{9}$ $\mathrm{CFU} /$ day can significantly reduce the triglycerides level by $22 \%$ in rats with high-fat diet while consumption of these bacteria at $10^{10} \mathrm{CFU} /$ day can decrease the level of triglycerides by $46 \%$ in highfructose-fed rats [31, 32]. There are several human studies that have indicated that the administration of probiotics has led to a decrease in serum total cholesterol, triglyceride, and LDL [33-35]. On the other hand, some clinical trial reports have shown that the consumption of some probiotics might have no significant effects on lipid profile [36, 37].

Numerous studies have shown probiotics may affect cholesterol metabolism through the regulation of genes involved in cholesterol biosynthesis pathway $[29,38,39]$.

In contrast to the findings of some studies, we observed a significant decrease in the expression of HMGCR gene in BS group compared to the HFD group. However, some studies have indicated that the administration of some probiotics such as $L$. plantarum, and Lactobacillus rhamnosus, can lead to up-regulation of HMGCR in Caco-2 cells and liver, respectively [38, 40, 41]. Interestingly, in vivo experiments have shown that taking cholesterollowering medicines (e.g., Ezetimibe and Simvastatin) elevates intestinal expression of HMGCR [42, 43].

Besides, our findings showed that $B$. subtilis JQ61816 cause a significant decrease in CYP7A1 expression in BS group. Our result is in agreement with Yadav et al. study, which has shown that consumption of milk fermented with two strains of $L$. rhamnosus can cause down-regulation of CYP7A1 in hypercholesterolemic rats [44]. However, our findings are in contrast to some studies which have shown probiotics consumption leads to overexpression of CYP7A1 [39, 45].

CYP7A1 gene is a part of bile acid-producing pathway which utilize cholesterol as its substrate, downregulation of this gene might occur for two reasons, in the first hypothesis, due to consumption of diet cholesterol by B. subtilis cholesterol oxidase [46], plasma cholesterol level could decrease, therefore due to depletion of CYP7A1 substrate this gene could be downregulated. In the second hypothesis, $B$. subtilis squalene synthase-like enzyme (YisP) can catalyze the formation of farnesol (FOH) from farnesyl diphosphate (FPP) [47]. Farnesol subsequently can activate farnesoid X receptor (FXR) [48]. Activation of this receptor would lead to downregulation of CYP7A1 [49] and also reported to has a triglyceride-lowering effect. In the current study, these effects were observed in BS group. On the other hand, FXR activation is shown to be effective in intestinal cholesterol excretion and hyperlipidemia attenuation [50]. It seems that the excretion of intestinal cholesterol may lead to a decrease in blood cholesterol levels. Elevation of the HMGCR gene expression in BS group might occur to compensate for this reduction by increasing cellular biosynthesis of cholesterol. The proposed hypotheses are depicted in Supplementary Figure 1.

Here, we are reporting that B. subtilis JQ61816 exerts cholesterol-lowering and triglycerides-lowering effects on the host through regulation of expression of genes involved in lipid metabolism. According to numerous studies, it seems probiotics exert their effects in various mechanisms and several factors are associated with the mechanisms, including dosage of probiotics, type of probiotics bacteria, gut microbiota composition, etc. We suggest that administration of different doses of B. subtilis JQ61816 in a long period of time for further studies. Among the limitations of the present study we make a suggestion according to the results as the mechanisms of hypocholesterolemic effects of probiotics are not clear yet. The period of time for using probiotics may have roles in its hypocholesterolemic effects. Moreover, it is possible that the administration of this bacterium with other probiotics and even prebiotics may elevate its hypocholesterolemic effects.

Our findings showed that the administration of $B$. subtilis JQ61816 can reduce total serum cholesterol, triglycerides, and LDL, while increasing HDL levels. Moreover, B. subtilis JQ61816 can reduce hepatic enzymes that are associated with liver disorders such as fatty liver. Also, the level of urea and uric acid were decreased due to administration of $B$. subtilis JQ61816. 


\section{Supplementary files}

Supplementary file 1.

\section{Author contributions}

All authors contributed equally to this manuscript and approved the final version of the manuscripts.

\section{Conflict of interests}

The authors declare that they have no conflicts of interest.

\section{Ethical declarations}

All procedures were performed in accordance with the guidelines of the Medical Ethics Committee of Islamic Azad University of Arak, Iran.

\section{Financial Support}

None to be declared.

\section{References}

1. Upadhyay RK. Emerging risk biomarkers in cardiovascular diseases and disorders. J Lipids. 2015; 2015:971453.

2. Benjamin EJ, Blaha MJ, Chiuve SE, Cushman M, Das SR, Deo $\mathrm{R}$, et al. Heart disease and stroke statistics-2017 update: a report from the American Heart Association. circulation. 2017; 135(10):e146-e603.

3. World Health Organization. Global status report on noncommunicable diseases 2014. Geneva: WHO; 2014. Available at:

http://apps.who.int/iris/bitstream/10665/148114/1/9789241564 854_eng.pdf

4. Levine GN, Keaney JF, Jr., Vita JA. Cholesterol reduction in cardiovascular disease. Clinical benefits and possible mechanisms. N Engl J Med. 1995; 332(8):512-21.

5. Stefanick ML, Mackey S, Sheehan M, Ellsworth N, Haskell WL, Wood PD. Effects of diet and exercise in men and postmenopausal women with low levels of HDL cholesterol and high levels of LDL cholesterol. N Engl J Med. 1998; 339(1):12-20.

6. Polychronopoulos E, Panagiotakos DB, Polystipioti A. Diet, lifestyle factors and hypercholesterolemia in elderly men and women from Cyprus. Lipids Health Dis. 2005; 4:17.

7. Last AR, Ference JD, Falleroni J. Pharmacologic treatment of hyperlipidemia. Am Fam Physician. 2011; 84(5):551-8.

8. Eghdamian B, Ghose K. Mode of action and adverse effects of lipid lowering drugs. Drugs Today (Barc). 1998; 34(11):943-56. 9. Godlee F. Adverse effects of statins. BMJ. 2014; 348:g3306.

10. Azad MAK, Sarker M, Wan D. Immunomodulatory Effects of Probiotics on Cytokine Profiles. Biomed Res Int. 2018; 2018:8063647.

11. Dehkohneh A, Jafari P, Fahimi H. Effects of probiotic Lactobacillus paracasei TD3 on moderation of cholesterol biosynthesis pathway in rats. Iran J Basic Med Sci. 2019; 22(9):1004-9.
12. Ooi LG, Liong MT. Cholesterol-lowering effects of probiotics and prebiotics: a review of in vivo and in vitro findings. Int $\mathrm{J}$ Mol Sci. 2010; 11(6):2499-522.

13. Miraghajani M, Zaghian N, Dehkohneh A, Mirlohi M, Ghiasvand R. Probiotic Soy Milk Consumption and Renal Function Among Type 2 Diabetic Patients with Nephropathy: a Randomized Controlled Clinical Trial. Probiotics Antimicrob Proteins. 2019; 11(1):124-32.

14. Rafter J. Probiotics and colon cancer. Best Pract Res Clin Gastroenterol. 2003; $17(5): 849-59$.

15. Hill C, Guarner F, Reid G, Gibson GR, Merenstein DJ, Pot B, et al. Expert consensus document. The International Scientific Association for Probiotics and Prebiotics consensus statement on the scope and appropriate use of the term probiotic. Nat Rev Gastroenterol Hepatol. 2014; 11(8):506-14.

16. Gielen S, Landmesser U. The Year in Cardiology 2013: cardiovascular disease prevention. Eur Heart J. 2014; 35(5):30712.

17. Huang Y, Zheng Y. The probiotic Lactobacillus acidophilus reduces cholesterol absorption through the down-regulation of Niemann-Pick C1-like 1 in Caco-2 cells. Br J Nutr. 2010; 103(4):473-8.

18. Thobari J, Gansevoort R, De Jong P, De Jong-van Den Berg L. The effect lipid lowering drugs statins on increasing risk of albuminuria and reduce of renal function: A cohort study. Drug Safety. 2008; 31(10): 885-960-.

19. Davies GR. Reversible dysphasia and statins. J Korean Med Sci. 2012; $27(4): 458-9$.

20. Shehata MG, El-Sahn MA, El Sohaimy SA, Youssef MM. Role and Mechanisms Lowering Cholesterol by Dietary of Probiotics and Prebiotics: A Review. J Appl Sci. 2019; 19(8):737-46.

21. Fernandez ML, Roy S, Vergara-Jimenez M. Resistant starch and cholestyramine have distinct effects on hepatic cholesterol metabolism in guinea pigs fed a hypercholesterolemic diet. Nutr Res. 2000; 20(6):837-49.

22. Gallaher CM, Munion J, Hesslink R, Jr., Wise J, Gallaher DD. Cholesterol reduction by glucomannan and chitosan is mediated by changes in cholesterol absorption and bile acid and fat excretion in rats. $J$ Nutr. 2000; 130(11):2753-9.

23. Lin Y, Meijer GW, Vermeer MA, Trautwein EA. Soy protein enhances the cholesterol-lowering effect of plant sterol esters in cholesterol-fed hamsters. J Nutr. 2004; 134(1):143-8.

24. Lambert JM, Bongers RS, de Vos WM, Kleerebezem M. Functional analysis of four bile salt hydrolase and penicillin acylase family members in Lactobacillus plantarum WCFS1. Appl Environ Microbiol. 2008; 74(15):4719-26.

25. Pereira DI, Gibson GR. Cholesterol assimilation by lactic acid bacteria and bifidobacteria isolated from the human gut. Appl Environ Microbiol. 2002; 68(9):4689-93.

26. Liong MT, Shah NP. Acid and bile tolerance and cholesterol removal ability of lactobacilli strains. J Dairy Sci. 2005; 88(1):5566.

27. De Preter V, Vanhoutte T, Huys G, Swings J, De Vuyst L, Rutgeerts $\mathrm{P}$, et al. Effects of Lactobacillus casei Shirota, Bifidobacterium breve, and oligofructose-enriched inulin on colonic nitrogen-protein metabolism in healthy humans. Am J Physiol Gastrointest Liver Physiol. 2007; 292(1):G358-68. 


\section{Dehkohneh et al.}

28. Kim KP, Rhee CH, Park HD. Degradation of cholesterol by Bacillus subtilis SFF34 isolated from Korean traditional fermented flatfish. Lett Appl Microbiol. 2002; 35(6):468-72.

29. Heo W, Lee ES, Cho HT, Kim JH, Lee JH, Yoon SM, et al. Lactobacillus plantarum LRCC 5273 isolated from Kimchi ameliorates diet-induced hypercholesterolemia in $\mathrm{C} 57 \mathrm{BL} / 6$ mice. Biosci Biotechnol Biochem. 2018; 82(11):1964-72.

30. Briand F, Sulpice T, Giammarinaro P, Roux X. Saccharomyces boulardii CNCM I-745 changes lipidemic profile and gut microbiota in a hamster hypercholesterolemic model. Benef Microbes. 2019; 10(5):555-67.

31. Choi ID, Kim SH, Jeong JW, Lee DE, Huh CS, Hong SS, et al. Triglyceride-Lowering Effects of Two Probiotics, Lactobacillus plantarum KY1032 and Lactobacillus curvatus HY7601, in a Rat Model of High-Fat Diet-Induced Hypertriglyceridemia. J Microbiol Biotechnol. 2016; 26(3):483-7.

32. Park DY, Ahn YT, Huh CS, McGregor RA, Choi MS. Dual probiotic strains suppress high fructose-induced metabolic syndrome. World J Gastroenterol. 2013; 19(2):274-83.

33. Naruszewicz M, Johansson ML, Zapolska-Downar D, Bukowska H. Effect of Lactobacillus plantarum 299v on cardiovascular disease risk factors in smokers. Am J Clin Nutr. 2002; 76(6):1249-55.

34. Ahn HY, Kim M, Chae JS, Ahn YT, Sim JH, Choi ID, et al. Supplementation with two probiotic strains, Lactobacillus curvatus HY7601 and Lactobacillus plantarum KY1032, reduces fasting triglycerides and enhances apolipoprotein $\mathrm{A}-\mathrm{V}$ levels in non-diabetic subjects with hypertriglyceridemia. Atherosclerosis. 2015; 241(2):649-56.

35. Bernini LJ, Simão AN, Alfieri DF, Lozovoy MA, Mari NL, de Souza CH, et al. Beneficial effects of Bifidobacterium lactis on lipid profile and cytokines in patients with metabolic syndrome: A randomized trial. Effects of probiotics on metabolic syndrome. Nutrition. 2016; 32(6):716-9.

36. Dong H, Rowland I, Thomas LV, Yaqoob P. Immunomodulatory effects of a probiotic drink containing Lactobacillus casei Shirota in healthy older volunteers. Eur J Nutr. 2013; 52(8):1853-63.

37. Ivey KL, Hodgson JM, Kerr DA, Thompson PL, Stojceski B, Prince RL. The effect of yoghurt and its probiotics on blood pressure and serum lipid profile; a randomised controlled trial. Nutr Metab Cardiovasc Dis. 2015; 25(1):46-51.

38. Park S, Kang J, Choi S, Park H, Hwang E, Kang YG, et al. Cholesterol-lowering effect of Lactobacillus rhamnosus BFE5264 and its influence on the gut microbiome and propionate level in a murine model. PLoS One. 2018; 13(8):e0203150.
39. Wang G, Huang W, Xia Y, Xiong Z, Ai L. Cholesterol-lowering potentials of Lactobacillus strain overexpression of bile salt hydrolase on high cholesterol diet-induced hypercholesterolemic mice. Food Funct. 2019; 10(3):1684-95.

40. Palaniyandi SA, Damodharan K, Suh JW, Yang SH. Probiotic Characterization of Cholesterol-Lowering Lactobacillus fermentum MJM60397. Probiotics Antimicrob Proteins. 2020; 12(3):1161-72.

41. Michael DR, Moss JW, Calvente DL, Garaiova I, Plummer SF, Ramji DP. Lactobacillus plantarum CUL66 can impact cholesterol homeostasis in Caco-2 enterocytes. Benef Microbes. 2016; 7(3):443-51.

42. Catry E, Pachikian BD, Salazar N, Neyrinck AM, Cani PD, Delzenne NM. Ezetimibe and simvastatin modulate gut microbiota and expression of genes related to cholesterol metabolism. Life Sci. 2015; 132:77-84.

43. Engelking LJ, McFarlane MR, Li CK, Liang G. Blockade of cholesterol absorption by ezetimibe reveals a complex homeostatic network in enterocytes. J Lipid Res. 2012; 53(7):1359-68.

44. Yadav R, Vij R, Kapila S, Khan SH, Kumar N, Meena S, et al. Milk fermented with probiotic strains Lactobacillus rhamnosus MTCC: 5957 and Lactobacillus rhamnosus MTCC: 5897 ameliorates the diet-induced hypercholesterolemia in rats. Ann Microbiol. 2019; 69(5):483-94.

45. Watanabe S, Katsube T, Hattori H, Sato H, Ishijima T, Nakai Y, et al. Effect of Lactobacillus brevis 119-2 isolated from Tsuda kabu red turnips on cholesterol levels in cholesterol-administered rats. $\mathrm{J}$ Biosci Bioeng. 2013; 116(1):45-51.

46. Kumari L, Kanwar SS. Purification and Characterization of an Extracellular Cholesterol Oxidase of Bacillus subtilis Isolated from Tiger Excreta. Appl Biochem Biotechnol. 2016; 178(2):353-67. 47. Feng X, Hu Y, Zheng Y, Zhu W, Li K, Huang CH, et al. Structural and functional analysis of Bacillus subtilis YisP reveals a role of its product in biofilm production. Chem Biol. 2014; 21(11):1557-63.

48. Forman BM, Goode E, Chen J, Oro AE, Bradley DJ, Perlmann $\mathrm{T}$, et al. Identification of a nuclear receptor that is activated by farnesol metabolites. Cell. 1995; 81(5):687-93.

49. Xu G, Li H, Pan LX, Shang Q, Honda A, Ananthanarayanan M, et al. FXR-mediated down-regulation of CYP7A1 dominates LXRalpha in long-term cholesterol-fed NZW rabbits. J Lipid Res. 2003; 44(10):1956-62.

50. Singh AB, Dong B, Kraemer FB, Liu J. FXR activation promotes intestinal cholesterol excretion and attenuates hyperlipidemia in SR-B1-deficient mice fed a high-fat and highcholesterol diet. Physiol Rep. 2020; 8(5):e14387. 